\title{
Successful treatment of a patient with Philadelphia chromosome-positive acute lymphoblastic leukemia complicated by bone marrow necrosis and acute renal insufficiency: A case report
}

\author{
JIEYUN XIA ${ }^{1}$, HAIYING SUN ${ }^{1}$, ZHILING YAN ${ }^{1}$, FENG ZHU $^{1}$, KAI ZHAO $^{1}$ and KAILIN XU ${ }^{1,2}$ \\ ${ }^{1}$ Department of Hematology, The Affiliated Hospital of Xuzhou Medical University; \\ ${ }^{2}$ Key Laboratory of Bone Marrow Stem Cell, Blood Diseases Institute, \\ Xuzhou Medical University, Xuzhou, Jiangsu 221002, P.R. China
}

Received April 18, 2018; Accepted June 19, 2018

DOI: $10.3892 / \mathrm{mco} .2018 .1661$

\begin{abstract}
Philadelphia chromosome (Ph)-positive acute lymphoblastic leukemia (ALL) complicated by bone marrow necrosis $(B M N)$ and acute renal insufficiency (ARI) is rare in clinical practice. The aim of the present study was to summarize the clinical characteristics and treatment methods of a case of $\mathrm{Ph}^{+}$ALL complicated by BMN and ARI. A 23-year-old male patient presented with pyrexia and a 2-month history of bone pain, and was diagnosed with $\mathrm{Ph}^{+}$ ALL complicated with BMN and ARI on peripheral blood tests, blood biochemistry tests, BM smear and fluorescence in situ hybridization. The patient underwent repeated hemodialysis, imatinib combination and maintenance chemotherapy, followed by allogeneic hematopoietic stem-cell transplantation. Some of the clinical signs and symptoms were alleviated, whereas others disappeared, and renal function was restored to normal. BM aspiration confirmed absence of necrosis and null lymphoblasts. Therefore, timely accurate diagnosis and effective treatment are crucial for patients with $\mathrm{Ph}^{+}$ALL exhibiting potentially fatal complications.
\end{abstract}

\section{Introduction}

Acute lymphoblastic leukemia (ALL) is a common type of clonal hematopoietic malignant disease of lymphocyte precursor cells (1). Clinical manifestations, such as anemia, bleeding, infection, as well as enlargement of the liver, spleen and lymph nodes, may occur due to the infiltration of tissues

Correspondence to: Professor Kailin Xu, Department of Hematology, The Affiliated Hospital of Xuzhou Medical University, 99 Huaihai Road, Xuzhou, Jiangsu 221002, P.R. China

E-mail: 1ihmd@163.com

Key words: acute lymphoblastic leukemia, bone marrow necrosis, acute renal insufficiency, imatinib, chemotherapy and organs throughout the body by leukemic cells. Bone marrow necrosis (BMN) and acute renal insufficiency (ARI) are rare and frequently fatal conditions, which may occur as a primary or secondary manifestation of ALL $(2,3)$. However, it is extremely rare for BMN and ARI to occur simultaneously in a patient with Philadelphia chromosome-positive $\left(\mathrm{Ph}^{+}\right) \mathrm{ALL}$ and, to the best of our knowledge, such a case has not been reported to date. It is documented that $\mathrm{Ph}^{+} \mathrm{ALL}$ is associated with at least a $10 \%$ reduction of primary complete remission by standard induction chemotherapy, and long-term prognosis remains poor compared with that of $\mathrm{Ph}^{-} \mathrm{ALL}$ (4).

We herein describe the case of a patient with $\mathrm{Ph}^{+} \mathrm{ALL}$ concomitant with BMN and ARI, who was treated with imatinib combination and maintenance therapy, allogeneic hematopoietic stem-cell transplantation (allo-HSCT), and various comprehensive treatments, such as repeated hemodialysis, anti-infective therapy and transfusion of platelets and erythrocyte suspension.

\section{Case report}

A 23-year-old male patient with paroxysmal bone pain and intermittent pyrexia for a duration of 2 months was referred to the Department of Hematology of the Affiliated Hospital of Xuzhou Medical University during April, 2015. Upon admission, the results of the emergency blood routine tests were as follows: White blood cell (WBC) count $41.8 \times 10^{9} / 1$, hemoglobin concentration $103 \mathrm{~g} / 1$ and platelet count $104 \times 10^{9} / 1$; the blast proportion was $21 \%$. The results of the blood biochemical tests were as follows: Serum creatinine ( $\mathrm{SCr}) 0.95 \mathrm{mg} / \mathrm{dl}$, blood urea nitrogen (BUN) $19.97 \mathrm{mg} / \mathrm{dl}$, uric acid $7.77 \mathrm{mg} / \mathrm{dl}$, calcium $9.58 \mathrm{mg} / \mathrm{dl}$, lactate dehydrogenase (LDH) $2263 \mathrm{U} / \mathrm{l}$ and alkaline phosphatase (ALP) $214 \mathrm{U} / 1$.

Following admission, the patient's condition deteriorated rapidly, with recurrent hyperpyrexia $\left(40.2^{\circ} \mathrm{C}\right)$, chills, nausea and vomiting. The patient also had severe systemic arthralgia and oliguria ( $200 \mathrm{ml} / 24 \mathrm{~h})$. Upon examination, tachypnea, tachycardia, ecchymoses and pitting oedema of the lower extremities were observed. Blood routine tests revealed a 
significant decline in the WBC count (from 41.8 to $11.7 \times 10^{9} / 1$ ), hemoglobin level $(95 \mathrm{~g} / \mathrm{l})$ and platelet count $\left(2 \times 10^{9} / 1\right)$. The results of the biochemical laboratory test were as follows: $\mathrm{SCr} 5.67 \mathrm{mg} / \mathrm{dl}$, BUN $86.35 \mathrm{mg} / \mathrm{dl}$, uric acid $18.31 \mathrm{mg} / \mathrm{dl}$, calcium $6.17 \mathrm{mg} / \mathrm{dl}$, phosphorus $10.31 \mathrm{mg} / \mathrm{dl}$, and an LDH level increased up to $3,778 \mathrm{U} / 1$. Abdominopelvic ultrasonography revealed normal-sized kidneys, with increased parenchymal echogenicity and absence of hydronephrosis, and a void bladder. For diagnosis, BM aspirate samples were obtained, which were brown in colour, and the cell morphology indicated BMN (Fig. 1A and B). A BM biopsy specimen revealed hypercellularity in lymphocytes, with $20 \%$ lymphoblasts. Flow cytometric immunophenotyping of BM cells revealed that 96.9\% were CD19-positive, $85.9 \%$ were CD10-positive, $47.2 \%$ were CD10/CD33-positive, $34.8 \%$ were CD20-positive, $90.6 \%$ were CD34/HLA-DR-positive, $2.2 \%$ were cCD79a-positive, and $46.2 \%$ were cCD22-positive. Fluorescence in situ hybridization (FISH) came back positive for the BCR-ABL fusion gene, and the BCR-ABL/ABL ratio was $82.82 \%$ (Fig. 2). Therefore, the patient was diagnosed with $\mathrm{Ph}^{+} \mathrm{ALL}$ (B-cell) complicated with BMN and ARI based on the medical history, clinical manifestations and laboratory test results.

Subsequent to admission, treatment was initiated, including antipyretic therapy, rehydration, energy replenishment [daily treatment with cytidine disodium triphosphate $(80 \mathrm{mg})$, inosine (400 mg), 50\% glucose (40 g), ascorbic acid (1 g) and insulin (16 units) for a total of 7 days] and oral tramadol for analgesia. On the third day after admission, prednisolone (600 mg/day), allopurinol and furosemide were administered, along with transfusion of platelets and erythrocyte suspension, hemodialysis with therapy for acid-base imbalance, electrolyte disorders, and anti-infection with imipenem. At 10 days after treatment initiation, the patient's clinical status had significantly improved, urine output gradually increased, the oedema of the lower extremities subsided, and the levels of SCr, BUN and uric acid had almost returned to normal; therefore, dialysis was terminated. Thereafter, the patient received cyclophosphamide (CY; $600 \mathrm{mg} /$ day) to reduce the tumor load, and prednisolone administration was continued at $400 \mathrm{mg} / \mathrm{day}$. On the 12th day after admission, the patient received imatinib combined with induction chemotherapy (imatinib plus VIP), with imatinib administered in combination with vindesine $(4 \mathrm{mg} /$ day $)$ on days $1,8,15$ and 22; idarubicin (10 mg/day) was administered for the first 3 consecutive days, with imatinib ( $400 \mathrm{mg} / \mathrm{day}$ ) and prednisolone $(60 \mathrm{mg} /$ day) administered daily. In addition, the patient continually received supportive treatment, including hydration, alkalization, hepatoprotection and renoprotection, energy replenishment as previously aforementioned and anti-infection treatment. On day 14 of chemotherapy, the VIP regimen was discontinued due to severe BM suppression, while transfusion of erythrocytes and platelets was performed, along with administration of recombinant granulocyte colony-stimulating factor to increase the WBC count. On the 42nd day of hospitalization, the results of routine blood tests had nearly returned to normal, a BM smear revealed no signs of necrosis and null lymphoblasts, indicating partial remission of the primary disease. Thereafter, the patient received the second course of chemotherapy (imatinib plus VDCLP), with imatinib (400 mg/day) administered daily in combination with vindesine (4 mg/day) on days $1,8,15$ and 22; daunorubicin
(40 mg/day) was administered on days $1-3$, CY (1.0 g/day) on days 1 and 15 , L-asparaginase $(10,000 \mathrm{U})$ on alternate days from day 10 to 22 and prednisolone $(60 \mathrm{mg} / \mathrm{day})$ from day 1 to 28 . Comprehensive treatments were also performed as described above, and the prednisolone dose was reduced gradually from day 15 onwards and discontinued on day 28 . On the 72nd day of hospital stay, the results of routine blood tests were normal for the three types of blood cells, BM examination revealed $0 \%$ lymphoblasts, and cytogenetic examination of BM cells revealed a normal karyotype and null BCR/ABL, indicating that hematological remission as well as complete remission at the genetic and molecular level had been achieved. Subsequently, single-agent imatinib was administered as maintenance chemotherapy at $400 \mathrm{mg} /$ day. Three weeks later, an intensified chemotherapy with high-dose methotrexate and vincristine + prednisone was administered, with methotrexate $3 \mathrm{~g}$ and vincristine $30 \mathrm{mg}$ on day 1 and prednisone (30 mg/day) on days 1-7. After the third course of chemotherapy, pretreatment chemotherapy with the improved busulfan (BU)/CY regimen (Ara-C $5.0 \mathrm{~g}$ on days -10 and -9 , BU $48 \mathrm{mg}$ q $6 \mathrm{~h} \times 3 \mathrm{~d}$ on days- 8 to $-6, \mathrm{CY} 28 \mathrm{mg}$ on days -5 and -4 , lomustine $450 \mathrm{mg}$ and anti-thymocyte globulin $240 \mathrm{mg}$ on days -5 to -2 ) was performed. During August 2015, the patient underwent allo-HSCT. One month after allo-HSCT, rapid and complete hematopoietic reconstitution was achieved, and the patient showed no major complications occurred. Repeated FISH tests revealed negative expression of the BCR-ABL fusion gene. A total of 5 months after allo-HSCT, treatment with imatinib (300 mg/day) was resumed in order to prevent relapse. Consider that leukemia can impair the central nervous system and periocular tissues, the patient was pretreated with the fludarabine and cyclophosphamide regimen [fludarabine phosphate (50 mg; once daily on days 1-3); and cyclophosphamide (1.2 g; once daily on days 1 and 2)] between April 6 and 8, 2017. Following this, chimeric antigen receptor (CAR)-T cells present in peripheral blood obtained from the sibling of the patient $\left(1.73 \times 10^{6} / \mathrm{kg}\right)$ were intravenously infused on April 11, 2017. During May 2017, the patient was discharged from hospital and was in remission. The final follow-up appointment was performed 2 years following initial diagnosis, and the patient remained in remission.

\section{Discussion}

BMN is characterized by coagulation, architectural destruction and necrosis of a large area of hematopoietic and stromal tissues within the BM $(5,6)$. In addition, high levels of LDH and ALP are common laboratory findings in the majority of cases with BMN $(5,7)$. Rather than the typical signs or symptoms of leukemia in the early stages, our patient presented with pyrexia and arthralgia, a progressive decrease in blood cell counts, and elevated LDH and ALP levels, all of which strongly suggested a diagnosis of BMN. Consequently, based on a series of tests, a diagnosis of $\mathrm{Ph}^{+}$ALL (B-cell) complicated with BMN was confirmed.

The mechanism underlying ALL as a contributor to BMN has not been fully elucidated. Routine blood tests upon hospital admission revealed elevated WBC count of $41.8 \times 10^{9} / 1$, and cytological examination of BM revealed elevation of the lymphocytic proportion (20\% lymphoblasts). 

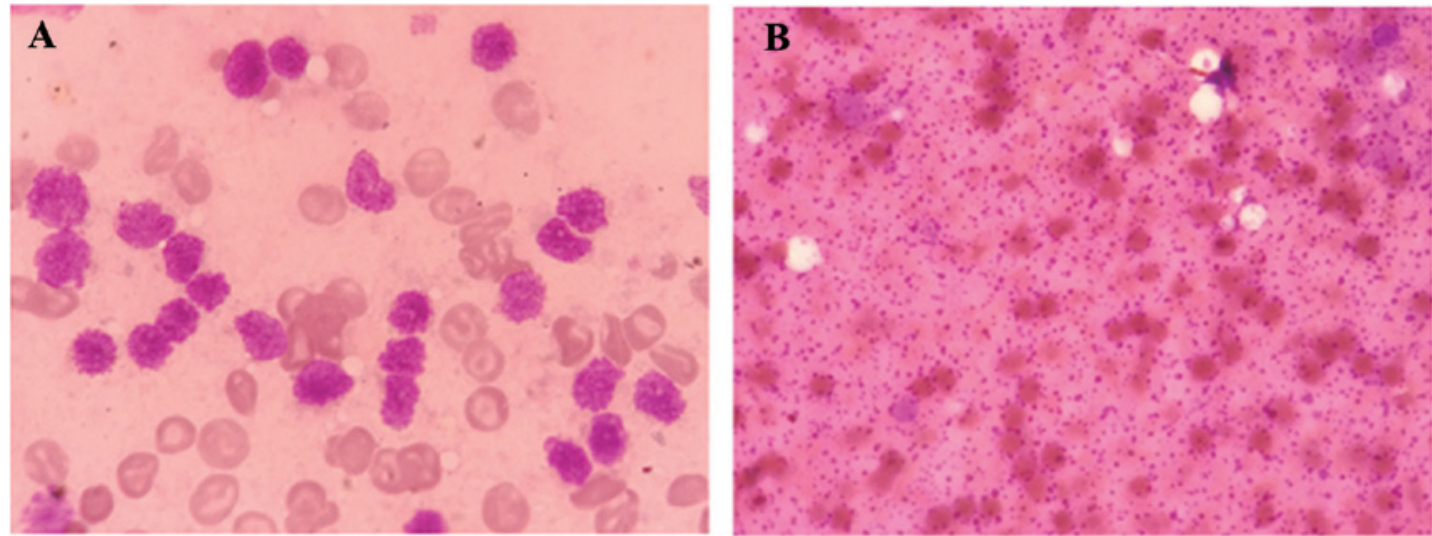

Figure 1. Bone marrow (smear) cell morphology (Wright staining). (A) Pre-treatment bone marrow smear revealed active proliferation of bone marrow cells, irregularly shaped granulocytes, intercellular purple-red nebulous amorphous substance, erythrocyte debris, increased number of lymphocytes with markedly abnormal morphology and an irregular contour of the cell body. (B) The cells were dissolved, exhibiting blurred edges and endolysis.

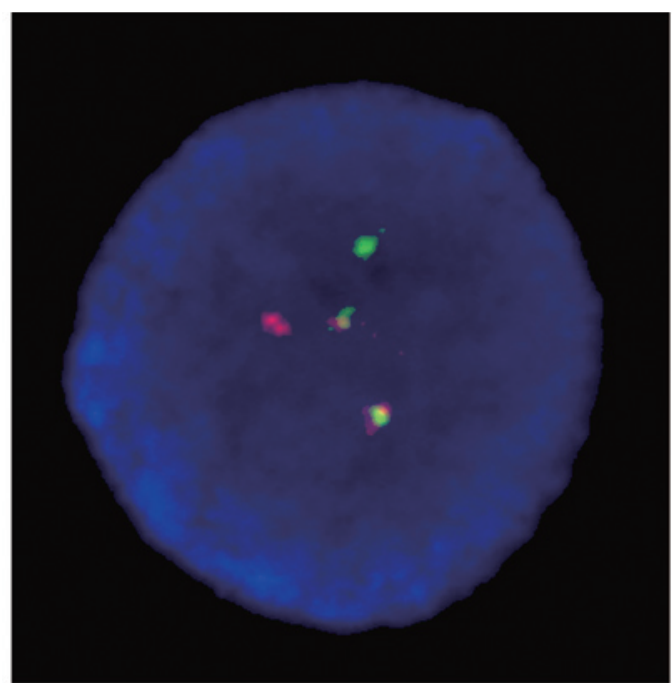

Figure 2. Detection of the BCR-ABL fusion gene in ALL by FISH prior to treatment. One green fluorescent marker (BCR gene), one red fluorescent marker (ABL gene), and two yellow fluorescent markers (BCR-ABL fusion gene) were observed. ALL, acute lymphocytic leukemia; FISH, fluorescence in situ hybridization.

Thus, BMN may be attributable to the microcirculatory disturbance due to an overload of lymphoblasts (8). The patient presented with symptoms of tumor necrosis factor (TNF)- $\alpha$ toxicity at early onset manifesting as hyperpyrexia, chills, headache, nausea and fatigue. Therefore, TNF- $\alpha$ may play a pivotal role in the pathogenesis of $\operatorname{BMN}(7,9)$. Since the patient received no special medication prior to the diagnosis of BMN, the possibility of BMN caused by drugs or poisons may be excluded.

ARI is defined as a sudden reduction in renal function, characterized by an absolute increase in serum SCr level to $\geq 0.3 \mathrm{mg} / \mathrm{dl}$ or by $50 \%$ compared with baseline (10). ARI is a common and serious complication associated with malignancy, which occurs in various clinical settings for numerous reasons (11-13). In the present case, at $32 \mathrm{~h}$ after admission, the patient experienced nausea, vomiting, oliguria and oedema in the bilateral lower extremities. Renal function test results revealed a SCr level of $5.67 \mathrm{mg} / \mathrm{dl}$ ( $>1.5$-fold from baseline, with an absolute increase of $4.48 \mathrm{mg} / \mathrm{dl},>0.3 \mathrm{mg} / \mathrm{dl}$ ), BUN
$86.35 \mathrm{mg} / \mathrm{dl}$ and uric acid $18.31 \mathrm{mg} / \mathrm{dl}$ prior to the induction of chemotherapy, indicating the development of ARI according to the relevant diagnostic criteria (10).

Despite the fact that the mechanisms through which ALL contributes to ARI are not clearly understood, ARI may be an important sequela of the tumor lysis syndrome (TLS). TLS is a term used to describe a series of metabolic abnormalities and multiple organ dysfunctions that result from the rapid release of the intracellular contents of lysed tumor cells. TLS is classified into laboratory and clinical TLS, with the former involving at least two abnormalities in serum uric acid, potassium, phosphorus or calcium levels (14). Clinical TLS is defined as the presence of laboratory TLS and one of the following: ARI, cardiac arrhythmias, or seizures. It has been reported that patients with ALL or non-Hodgkin lymphoma have a high incidence of TLS, and that TLS may be the leading cause of ARI in such patients $(15,16)$. Additional univariate analysis revealed that TLS develops more frequently in patients with elevated serum uric acid, SCr, LDH and WBC count $(17,18)$. In the present case, the patient fit the criteria of TLS with respect to 3 items of laboratory results (hyperuricemia $>8.0 \mathrm{mg} / \mathrm{dl}$, hyperphosphatemia $>4.6 \mathrm{mg} / \mathrm{dl}$ and hypocalcemia $<7.0 \mathrm{mg} / \mathrm{dl}$ ) and one clinical symptom (ARI), accompanied by high-risk characteristics, namely increased LDH level and WBC count; therefore, our patient presented with clinical TLS (14).

The pathogenesis of ARI resulting from TLS is complex and multifactorial. Of the numerous factors that can cause ARI, uric acid and phosphorus are the most common. Hyperuricemia is the main characteristic of TLS metabolic disturbance, which results from the breakdown of purine-containing nucleic acids due to lysed tumor cells. Previous studies demonstrated that hyperuricemia plays a major role in the pathophysiological process of ARI associated with TLS $(19,20)$. In addition, new evidence suggests that uric acid may also cause ARI through a crystallopathy-independent mechanism observed in TLS, such as renal vasoconstriction, renal ischemia, oxidation and inflammation (21). Similarly, as a result of massive release of intracellular phosphate stores, hyperphosphatemia is also characteristic of TLS, and the accumulation of calcium phosphate in the renal tubules may also be involved in the pathogenesis of ARI in patients 
with TLS (22). Our patient exhibited a persistent elevation of blood uric acid and phosphorus levels and decreased calcium levels. Therefore, hyperuricemia and hyperphosphatemia may account for TLS-induced ARI in this patient. A case of leukemic infiltration into the kidneys presenting as ARI has been reported $(23,24)$. Unfortunately, our patient refused to undergo renal biopsy; thus, it remains uncertain whether leukemic cell infiltration was associated with ARI development.

Intriguingly, despite the low complete remission rates and shortened remission and disease-free survival duration in $\mathrm{Ph}^{+}$ ALL patients by conventional chemotherapy, a recent study demonstrated that imatinib can improve the complete remission rate at the early stage, prolong disease-free survival, and improve the prognosis of $\mathrm{Ph}^{+}$ALL patients (25). The present case further supports that imatinib may be a key treatment component for patients with $\mathrm{Ph}^{+} \mathrm{ALL}$ with potentially fatal complications, which may improve the conditions for subsequent allo-HSCT, even if this assertion has yet to be supported by more studies.

In summary, the main conclusions of this case study are as follows: First, in a patient with BMN and/or ARI, a diagnosis of ALL should be considered; and second, $\mathrm{Ph}^{+}$ALL complicated by BMN and ARI is a rare and serious condition, and timely accurate diagnosis and effective management are crucial for successful treatment and for improving the quality of life of such patients.

\section{Acknowledgements}

Not applicable.

\section{Funding}

No funding was received.

\section{Availability of data and materials}

Not applicable.

\section{Ethics approval and consent to participate}

Not applicable.

\section{Patient consent for publication}

The patient provided written informed consent for the publication of clinical data.

\section{Authors' contributions}

$\mathrm{JX}$ and $\mathrm{KX}$ were responsible for the data analysis and interpretation. HS, ZY, KZ and FZ collected scientific literature and clinical information. JX and FZ wrote and edited the manuscript. All authors have read and approved of the final version of this manuscript.

\section{Competing interests}

The authors declare that they have no competing interests.

\section{References}

1. Davi F, Gocke C, Smith S and Sklar J: Lymphocytic progenitor cell origin and clonal evolution of human B-lineage acute lymphoblastic leukemia. Blood 88: 609-621, 1996.

2. Montemayor-Montoya JL, De León-Cantú RE, Gómez-Almaguer D and Herrera-Garza JL: 3 cases of bone marrow necrosis in acute leukemia. Rev Invest Clin 49: 295-298, 1997 (In Spanish).

3. Canet E, Zafrani L, Lambert J, Thieblemont C, Galicier L, Schnell D, Raffoux E, Lengline E, Chevret S, Darmon M and Azoulay E: Acute kidney injury in patients with newly diagnosed high-grade hematological malignancies: Impact on remission and survival. PLoS One 8: e55870, 2013.

4. Moorman AV, Harrison CJ, Buck GA, Richards SM, Secker-Walker LM, Martineau M, Vance GH, Cherry AM, Higgins RR, Fielding AK, et al: Karyotype is an independent prognostic factor in adult acute lymphoblastic leukemia (ALL): Analysis of cytogenetic data from patients treated on the Medical Research Council (MRC) UKALLXII/Eastern Cooperative Oncology Group (ECOG) 2993 trial. Blood 109: 3189-3197, 2007.

5. Bernard C, Sick H, Boilletot A and Oberling F: Bone marrow necrosis. acute microcirculation failure in myelomonocytic leukemia. Arch Intern Med 138: 1567-1569, 1978.

6. Paydas S, Ergin M, Baslamisli F, Yavuz S, Zorludemir S, Sahin B and Bolat FA: Bone marrow necrosis: Clinicopathologic analysis of 20 cases and review of the literature. Am J Hematol 70: 300-305, 2002.

7. Lackner H, Strenger V, Sovinz P, Beham-Schmid C, Pilhatsch A, Benesch M, Schwinger W, Ulreich R, Schmidt S and Urban C: Bone marrow necrosis in a girl with Hodgkin's disease. Support Care Cancer 20: 2231-2234, 2012.

8. Moritake H, Obara M, Sameshima N, Asada Y, Komatsu H, Hyakuna N, Sugita K, Ishida Y, Kato M, Tanizawa A, et al: Analysis of the molecular mechanism underlying bone marrow necrosis with acute lymphoblastic leukemia. Int J Hematol 102: 349-356, 2015.

9. Seki Y, Koike T, Yano M, Aoki S, Hiratsuka M, Fuse I and Aizawa Y: Bone marrow necrosis with dyspnea in a patient with malignant lymphoma and plasma levels of thrombomodulin, tumor necrosis factor-alpha, and D-dimer. Am J Hematol 70: 250-253, 2002.

10. Kellum JA, Lameire N, Aspelin P, Barsoum RS, Burdmann EA, Goldstein SL, Herzog CA, Joannidis M, Kribben A, Levey AS, et al: Kidney Disease: Improving Global Outcomes (KDIGO) Acute Kidney Injury Work Group: KDIGO clinical practice guideline for acute kidney injury. Kidney Int Suppl 2: $1-138,2012$.

11. Abu-Alfa AK and Younes A: Tumor lysis syndrome and acute kidney injury: Evaluation, prevention, and management. Am J Kidney Dis 55 (5 Suppl 3): S1-S13; quiz S14-S19, 2010.

12. Soares M, Salluh JI, Carvalho MS, Darmon M, Rocco JR and Spector N: Prognosis of critically ill patients with cancer and acute renal dysfunction. J Clin Oncol 24: 4003-4010, 2006.

13. Canet E, Vincent F, Darmon M and Soares M: Acute kidney injury in hematological patients. Curr Opin Crit Care 21: 549-558, 2015.

14. Cairo MS and Bishop M: Tumour lysis syndrome: New therapeutic strategies and classification. Br J Haematol 127: 3-11, 2004.

15. Stapleton FB, Strother DR, Roy S III, Wyatt RJ, McKay CP and Murphy SB: Acute renal failure at onset of therapy for advanced stage Burkitt lymphoma and B cell acute lymphoblastic lymphoma. Pediatrics 82: 863-869, 1988.

16. Annemans L, Moeremans K, Lamotte M, Garcia Conde J, van den Berg H, Myint H, Pieters R and Uyttebroeck A: Incidence, medical resource utilisation and costs of hyperuricemia and tumour lysis syndrome in patients with acute leukaemia and non-Hodgkin's lymphoma in four European countries. Leuk Lymphoma 44: 77-83, 2003.

17. Coiffier B, Altman A, Pui CH, Younes A and Cairo MS: Guidelines for the management of pediatric and adult tumor lysis syndrome: An evidence-based review. J Clin Oncol 26: 2767-2778, 2008.

18. Oiwa K, Morita M, Kishi S, Okura M, Tasaki T, Matsuda Y, Tai K, Hosono N, Ueda T and Yamauchi T: High risk of tumor lysis syndrome in symptomatic patients with multiple myeloma with renal dysfunction treated with bortezomib. Anticancer Res 36: 6655-6662, 2016. 
19. Kjellstrand CM, Cambell DC II, von Hartitzsch B and Buselmeier TJ: Hyperuricemic acute renal failure. Arch Intern Med 133: 349-359, 1974

20. Tsokos GC, Balow JE, Spiegel RJ and Magrath IT: Renal and metabolic complications of undifferentiated and lymphoblastic lymphomas. Medicine (Baltimore) 60: 218-229, 1981.

21. Shimada M, Johnson RJ, May WS Jr, Lingegowda V, Sood P, Nakagawa T, Van QC, Dass B and Ejaz AA: A novel role for uric acid in acute kidney injury associated with tumour lysis syndrome. Nephrol Dial Transplant 24: 2960-2964, 2009.

22. Prasada H: Sevelamer hydrochloride for tumor lysis syndrome-related hyperphosphatemia. Indian Pediatr 52: 613-615, 2015.
23. Uprety D, Peterson A and Shah BK: Renal failure secondary to leukemic infiltration of kidneys in CLL-a case report and review of literature. Ann Hematol 92: 271-273, 2013

24. Spapen J, Fostier K, De Raeve H, Janssens P and Spapen H: An unexpected complication of chronic myelomonocytic leukemia: Severe renal failure due to malignant tubulo-interstitial cell infiltration. Int J Nephrol Renovasc Dis 9: 1-4, 2015.

25. Lim SN, Joo YD, Lee KH, Kim DY, Lee JH, Lee JH, Chi HS, Yun SC, Lee WS, Lee SM, et al: Long-term follow-up of imatinib plus combination chemotherapy in patients with newly diagnosed Philadelphia chromosome-positive acute lymphoblastic leukemia. Am J Hematol 90: 1013-1020, 2015. 